\title{
On positivity and base loci of vector bundles
}

\author{
Thomas Bauer • Sándor J. Kovács • Alex Küronya • \\ Ernesto C. Mistretta - Tomasz Szemberg - Stefano Urbinati
}

Received: 18 September 2014 / Revised: 16 January 2015 / Accepted: 26 January 2015 / Published online: 31 March 2015

(C) Springer International Publishing AG 2015

\begin{abstract}
The aim of this note is to shed some light on the relationships among some notions of positivity for vector bundles that arose in recent decades. Our purpose is to study several of the positivity notions studied for vector bundles with some notions of asymptotic base loci that can be defined on the variety itself, rather than on the projectivization of the given vector bundle. We relate some of the different notions conjectured to be equivalent with the help of these base loci, and we show that these can help simplify the various relationships between the positivity properties present in the literature. In particular, we define augmented and restricted base loci $\mathbb{B}_{+}(E)$ and $\mathbb{B}_{-}(E)$ of a vector bundle $E$ on the variety $X$, as generalizations of the corresponding
\end{abstract}

TB was partially supported by DFG Grant BA 1559/6-1. SJK was supported in part by NSF Grant DMS-1301888, by a Simons Fellowship, Grant \#304043, and by the Craig McKibben and Sarah Merner Endowed Professorship in Mathematics at the University of Washington. AK was supported in part by the DFG-Forschergruppe 790 Classification of Algebraic Surfaces and Compact Complex Manifolds, and the OTKA Grants 77476 and 81203 by the Hungarian Academy of Sciences. ECM and SU were partially supported by Cariparo Eccellenza Grant Differential methods in Arithmetic, Geometry and Algebra. TSz was partially supported by NCN Grant UMO-2011/01/B/ST1/04875. SU was partially supported by Grant CPDA122824/12, Padova University.

T. Bauer

Fachbereich Mathematik und Informatik, Philipps-Universität Marburg, Hans-Meerwein-Straße, Lahnberge, 35032 Marburg, Germany

e-mail: tbauer@mathematik.uni-marburg.de

\section{S. J. Kovács}

Department of Mathematics, University of Washington, Seattle, WA 98195, USA

e-mail: skovacs@uw.edu

\section{A. Küronya}

Mathematics Institute, Budapest University of Technology and Economics,

Egry József u. 1, Budapest 1111, Hungary

e-mail: alex.kuronya@math.bme.hu 
notions studied extensively for line bundles. As it turns out, the asymptotic base loci defined here behave well with respect to the natural map induced by the projectivization of the vector bundle $E$.

Keywords Vector bundles $\cdot$ Positivity $\cdot$ Base loci

Mathematics Subject Classification $14 \mathrm{C} 20 \cdot 14 \mathrm{D} 20 \cdot 14 \mathrm{~J} 60$

\section{Introduction}

The aim of this note is to shed some light on the relationships among some notions of positivity for vector bundles that arose in recent decades.

Positivity properties of line bundles have long played a major role in projective geometry; they have once again become a center of attention recently, mainly in relation with advances in birational geometry, especially in the framework of the Minimal Model Program. Positivity of line bundles has often been studied in conjunction with numerical invariants and various kinds of asymptotic base loci, see for example [2,5].

At the same time, many positivity notions have been introduced for vector bundles of higher rank, generalizing some of the properties that hold for line bundles. While the situation in rank one is well-understood, at least as far as the interdepencies between the various positivity concepts is concerned, we are quite far from an analogous state of affairs for vector bundles in general.

In an attempt to generalize bigness for the higher rank case, some positivity properties have been put forward by Viehweg (in the study of fibrations in curves [18]), and Miyaoka (in the context of surfaces [14]), and are known to be different from the generalization given by using the tautological line bundle on the projectivization of the considered vector bundle, cf. [11]. The differences between the various definitions of bigness are already present in the works of Lang concerning the Green-Griffiths conjecture, see [10].

Our purpose is to study several of the positivity notions studied for vector bundles with the help of asymptotic base loci that can be defined on the variety itself, rather than on the projectivization of the given vector bundle. We relate some of the different notions conjectured to be equivalent with the help of these base loci, and we show that these can help simplify the various relationships between the positivity properties present in the literature.

E. C. Mistretta $(\bowtie) \cdot$ S. Urbinati

Dipartimento di Matematica, Università degli Studi di Padova,

Via Trieste 63, 35121 Padova, Italy

e-mail: ernesto@math.unipd.it

S. Urbinati

e-mail: urbinati.st@gmail.com

T. Szemberg

Pedagogical Univarsity Cracow, Podchorążych 2, 30-084 Kraków, Poland

e-mail: tomasz.szemberg@gmail.com 
In particular, we define augmented and restricted base loci $\mathbb{B}_{+}(E)$ and $\mathbb{B}_{-}(E)$ of a vector bundle $E$ on the variety $X$, as generalizations of the corresponding notions studied extensively for line bundles. As it turns out, the asymptotic base loci defined here behave well with respect to the natural map induced by the projectivization of the vector bundle $E$, as shown in Sect. 3 .

The relationship between these base loci with the positivity notions appearing in the literature goes as follows.

Theorem 1.1 Let $X$ be a smooth projective variety and $E$ a vector bundle on $X$. Then:

(1.1.1) $E$ is ample if and only if $\mathbb{B}_{+}(E)=\emptyset$;

(1.1.2) $E$ is nef if and only if $\mathbb{B}_{-}(E)=\emptyset$;

(1.1.3) $E$ is pseudo-effective if and only if $\mathbb{B}_{-}(E) \neq X$;

(1.1.4) $E$ is weakly positive if and only if $\overline{\mathbb{B}_{-}(E)} \neq X$, see Sect. 5;

(1.1.5) $E$ is V-big if and only if $\mathbb{B}_{+}(E) \neq X$, see Sect. 6;

(1.1.6) Assume that $E$ is a nef vector bundle. Then $E$ is almost everywhere ample if and only if $\mathbb{B}_{+}(E) \neq X$, cf. Sect. 8 .

The paper is organized as follows: in Sects. 2 and 3 we give the definition and basic properties of the asymptotic base loci for vector bundles, and relate these loci with the ones on the projectivizations. In Sect. 4 we recall the various positivity properties for line bundles and their relationship with asymptotic base loci. Section 5 is devoted to a study of positivity properties of vector bundles related to the restricted base locus, while Sect. 6 is given over to an investigation of connection between positivity properties of vector bundles related and augmented base loci. In Sects. 7 and 8 we study almost everywhere ampleness and relate it to V-bigness.

\section{Definitions and first properties}

Convention Throughout the paper we are working with vector bundles of finite rank, but for various reasons we find it more convenient to work with the associated sheaf of sections which is a locally free coherent $\mathcal{O}_{X}$-module. We will follow the usual abuse of terminology and while exclusively using this associated sheaf, we will still call it a vector bundle. If, rarely, we want to refer to a vector bundle and mean a vector bundle we will call it the total space of the vector bundle. We will also work with line bundles, which of course refers to a locally free sheaf of rank 1 . For a line bundle $L$ we will denote by $c_{1}(L)$ the associated Weil divisor on $X$.

With that convention fixed we are making the following notation that we will use through the entire paper.

Notation Let $X$ be a smooth projective variety over the complex numbers, and $E$ a vector bundle (i.e., according to the convention really a locally free sheaf) over $X$. For a point $x \in X, E_{x}=E \otimes_{\mathcal{O}_{X}} \mathcal{O}_{X, x}$ denotes the stalk of $E$ at the point $x$ and $E(x)=E \otimes_{\mathcal{O}_{X}} \kappa(x)$ where $\kappa(x)$ is the residue field at $x$. Clearly, $E(x)$ is the fiber of the total space of $E$ over the point $x$. In particular, $E(x)$ is a vector space of dimension $r=\operatorname{rk} E$. 
Definition 2.1 We define the base locus of $E$ (over $X$ ) as the subset

$$
\operatorname{Bs}(E)=\left\{x \in X: H^{0}(X, E) \rightarrow E(x) \text { is not surjective }\right\},
$$

and the stable base locus of $E$ (over $X$ ) as

$$
\mathbb{B}(E)=\bigcap_{m>0} \operatorname{Bs}\left(\operatorname{Sym}^{m} E\right) .
$$

Remark 2.2 The assertions below follow immediately from the definition:

(2.2.1) As $\operatorname{Bs}(E)=\operatorname{Bs}\left(\operatorname{Im}\left(\bigwedge^{\mathrm{rk} E} H^{0}(X, E) \rightarrow H^{0}(X\right.\right.$, det $\left.\left.E)\right)\right)$, these loci are closed subsets, and carry a natural scheme structure.

(2.2.2) For any positive integer $c>0, \mathbb{B}(E)=\mathbb{B}\left(\operatorname{Sym}^{c} E\right)$, and the same holds for $\mathbb{B}_{-}$and $\mathbb{B}_{+}$defined below.

Remark 2.3 The rank of the natural linear map $H^{0}(X, E) \rightarrow E(x)$ induces a stratification of $X$ into locally closed subsets.

Definition 2.4 Let $r=p / q \in \mathbb{Q}_{>0}$ be a positive rational number, and $A$ a line bundle on $X$. We will use the following notation:

$$
\mathbb{B}(E+r A)=\mathbb{B}\left(\operatorname{Sym}^{q} E \otimes A^{p}\right), \quad \mathbb{B}(E-r A)=\mathbb{B}\left(\operatorname{Sym}^{q} E \otimes A^{-p}\right) .
$$

Note that if $r=p^{\prime} / q^{\prime}$ is another representation of $r$ as a fraction, then $q^{\prime} p=p^{\prime} q$, hence

$$
\begin{aligned}
\operatorname{Sym}^{q^{\prime}}\left(\operatorname{Sym}^{q} E \otimes A^{p}\right) \simeq \operatorname{Sym}^{q^{\prime} q} E \otimes A^{q^{\prime} p} & \simeq \operatorname{Sym}^{q^{\prime} q} E \otimes A^{p^{\prime} q} \\
& \simeq \operatorname{Sym}^{q}\left(\operatorname{Sym}^{q^{\prime}} E \otimes A^{p^{\prime}}\right),
\end{aligned}
$$

and therefore, by (2.2.2), $\mathbb{B}\left(\operatorname{Sym}^{q} E \otimes A^{p}\right)=\mathbb{B}\left(\operatorname{Sym}^{q^{\prime}} E \otimes A^{p^{\prime}}\right)$ and hence $\mathbb{B}(E+r A)$ is well-defined. A similar argument shows that $\mathbb{B}(E-r A)$ is also well-defined.

Let $A$ be an ample line bundle on $X$, we define the augmented base locus of $E$ as

$$
\mathbb{B}_{+}^{A}(E)=\bigcap_{r \in \mathbb{Q}^{>0}} \mathbb{B}(E-r A),
$$

and the restricted base locus of $E$ as

$$
\mathbb{B}_{-}^{A}(E)=\bigcup_{r \in \mathbb{Q}^{>0}} \mathbb{B}(E+r A) .
$$

Remark 2.5 The definitions above yield the following properties:

(2.5.1) The loci $\mathbb{B}_{+}^{A}(E)$ and $\mathbb{B}_{-}^{A}(E)$ do not depend on the choice of the ample line bundle $A$, so we can write $\mathbb{B}_{+}(E)$ and $\mathbb{B}_{-}(E)$ for the augmented and restricted base locus of $E$, respectively. 
(2.5.2) For any $r_{1}>r_{2}>0$ we have $\mathbb{B}\left(E+r_{1} A\right) \subseteq \mathbb{B}\left(E+r_{2} A\right)$ and $\mathbb{B}\left(E-r_{2} A\right) \subseteq$ $\mathbb{B}\left(E-r_{1} A\right)$.

(2.5.3) In particular, for any $\varepsilon>0$ we have $\mathbb{B}(E+\varepsilon A) \subseteq \mathbb{B}(E) \subseteq \mathbb{B}(E-\varepsilon A)$.

(2.5.4) Therefore we have

$$
\mathbb{B}_{+}(E)=\bigcap_{q \in \mathbb{N}} \mathbb{B}\left(E-\frac{1}{q} A\right), \quad \mathbb{B}_{-}(E)=\bigcup_{q \in \mathbb{N}} \mathbb{B}\left(E+\frac{1}{q} A\right) .
$$

(2.5.5) It follows that $\mathbb{B}_{+}(E)$ is closed, but even for line bundles, the locus $\mathbb{B}_{-}(E)$ is not closed in general: Lesieutre [13] proved that this locus can be a proper dense subset of $X$, or a proper dense subset of a divisor of $X$.

\section{Asymptotic invariants for vector bundles}

In the following sections we will relate augmented and restricted base loci for vector bundles to various positivity notions found in the literature. In order to achieve a better understanding of these positivity properties and the relations between them, it is necessary to investigate the dependence of asymptotic base loci for vector bundles, and the corresponding loci of the tautological quotient line bundles on the appropriate projectivizations.

Let $E$ be a vector bundle on a smooth projective variety $X, \pi: \mathbb{P}(E) \rightarrow X$ the projective bundle of rank one quotients of $E$, and $\mathcal{O}_{\mathbb{P}(E)}(1)$ the universal quotient of $\pi^{*} E$ on $\mathbb{P}(E)$. Then we immediately have

$$
\pi\left(\mathbb{B}\left(\mathcal{O}_{\mathbb{P}(E)}(1)\right)\right) \subseteq \mathbb{B}(E)
$$

In fact, if the evaluation map $H^{0}(X, E) \otimes \mathcal{O}_{X} \rightarrow E$ is surjective over a point $x \in X$, then the map

$$
H^{0}\left(\mathbb{P}(E), \mathcal{O}_{\mathbb{P}(E)}(1)\right) \otimes \mathcal{O}_{\mathbb{P}(E)}=H^{0}(X, E) \otimes \mathcal{O}_{\mathbb{P}(E)} \rightarrow \pi^{*} E \rightarrow \mathcal{O}_{\mathbb{P}(E)}(1)
$$

is surjective over any point $y \in \mathbb{P}(E)$ such that $\pi(y)=x$, and a similar argument applies to $\operatorname{Sym}^{m} E$.

More precisely, we have $\pi\left(\operatorname{Bs}\left(\mathcal{O}_{\mathbb{P}(E)}(1)\right)\right)=\operatorname{Bs}(E)$ : if a point $x \in X$ does lie in $\operatorname{Bs}(E)$, then the image of the map $H^{0}(X, E) \rightarrow E(x)$ is contained in some hyperplane $H \subset E(x)$, where the hyperplane $H$ corresponds to a point $y \in \pi^{-1}(x)$ contained in $\operatorname{Bs}\left(\mathcal{O}_{\mathbb{P}(E)}(1)\right)$.

It is not clear whether the inclusion $\pi\left(\mathbb{B}\left(\mathcal{O}_{\mathbb{P}(E)}(1)\right)\right) \subseteq \mathbb{B}(E)$ of stable loci is strict in general. However, as we will show right below, some useful connections rely on properties of augmented and restricted base loci, which exhibit a more predictable behavior with respect to the map $\pi$.

Proposition 3.1 Let $E$ be a vector bundle on a smooth projective variety $X$, $\pi: \mathbb{P}(E) \rightarrow X$ the projective bundle of one dimensional quotients of $E$, and $\mathcal{O}_{\mathbb{P}(E)}(1)$ the universal quotient of $\pi^{*} E$ on $\mathbb{P}(E)$. Then 


$$
\pi\left(\mathbb{B}_{-}\left(\mathcal{O}_{\mathbb{P}(E)}(1)\right)\right)=\mathbb{B}_{-}(E) .
$$

Proof Let us fix $H \in \operatorname{Pic}(X)$, a sufficiently ample line bundle such that $A=$ $\mathcal{O}_{\mathbb{P}(E)}(1) \otimes \pi^{*} H$ is very ample on $\mathbb{P}(E)$. Then

$$
\mathbb{B}_{-}\left(\mathcal{O}_{\mathbb{P}(E)}(1)\right)=\bigcup_{a \in \mathbb{N}} \mathbb{B}\left(\mathcal{O}_{\mathbb{P}(E)}(a) \otimes A\right)=\bigcup_{a \in \mathbb{N}} \bigcap_{b \in \mathbb{N}} \operatorname{Bs}\left(\mathcal{O}_{\mathbb{P}(E)}(a b) \otimes A^{b}\right)
$$

and

$$
\mathbb{B}_{-}(E)=\bigcup_{a \in \mathbb{N}} \bigcap_{b \in \mathbb{N}} \operatorname{Bs}\left(\operatorname{Sym}^{a b} E \otimes H^{b}\right) .
$$

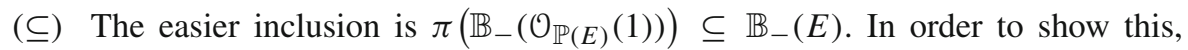
suppose that $x \in X$ and that $x \notin \mathbb{B}_{-}(E)$. Then for any integer $a>0$ there exists $b>0$ such that the vector bundle $\operatorname{Sym}^{a b} E \otimes H^{b}$ is generated by its global sections at $x \in X$. Then for all $a>0$ the line bundle $\mathcal{O}(2(a-1) b) \otimes A^{2 b}=\mathcal{O}(2 a b) \otimes \pi^{*} H^{2 b}$ which is a quotient of $\pi^{*}\left(\mathrm{Sym}^{2 a b} E \otimes H^{2 b}\right)$ is generated by its global sections (defined over the whole space $\mathbb{P}(E))$ on any point of the fibre $\pi^{-1}(x)$, so the fibre $\pi^{-1}(x)$ is contained in the complement of $\mathbb{B}_{-}\left(\mathcal{O}_{\mathbb{P}(E)}(1)\right)$.

(つ) Let us show now that $\pi\left(\mathbb{B}_{-}\left(\mathcal{O}_{\mathbb{P}(E)}(1)\right)\right) \supseteq \mathbb{B}_{-}(E)$ : Let $x \in X$ be a point such that $x \notin \pi\left(\mathbb{B}_{-}\left(\mathcal{O}_{\mathbb{P}(E)}(1)\right)\right)$. Then for any $a>0$ there exists $b>0$ such that $\mathcal{O}_{\mathbb{P}(E)}(2(a-1) b) \otimes A^{b}$ is generated on any point $y \in \pi^{-1}(x)$ by its global sections (defined on the whole $\mathbb{P}(E)$ ).

Then the line bundle

$$
\begin{aligned}
L=\mathcal{O}_{\mathbb{P}(E)}(2 a b) \otimes \pi^{*} H^{2 b} & \simeq \mathcal{O}_{\mathbb{P}(E)}(2(a-1) b) \otimes \mathcal{O}_{\mathbb{P}(E)}(b) \otimes \pi^{*} H^{b} \\
& \otimes \mathcal{O}_{\mathbb{P}(E)}(b) \otimes \pi^{*} H^{b} \\
& \simeq\left(\mathcal{O}_{\mathbb{P}(E)}(2(a-1) b) \otimes A^{b}\right) \otimes A^{b}
\end{aligned}
$$

is the product of a line bundle which is generated by global sections (on $\mathbb{P}(E)$ ) at any point of the fiber $Y=\pi^{-1}(x)=\mathbb{P}(E(x))$ with a very ample line bundle, so its global sections (on $\mathbb{P}(E)$ ) define a closed immersion of $Y$ into a projective space. In other words, the linear system $H^{0}(\mathbb{P}(E), L)$ defines a rational map $\varphi: \mathbb{P}(E) \rightarrow$ $\mathbb{P}\left(H^{0}(\mathbb{P}(\mathbb{E}), L)\right)=\mathbb{P}^{N}$ which is a regular immersion on $Y$. Then in particular, for $m \gg 0$ the multiplication map $\operatorname{Sym}^{m} H^{0}(\mathbb{P}(\mathbb{E}), L) \rightarrow H^{0}\left(Y,\left.L^{m}\right|_{Y}\right)$ is surjective.

It follows that the map $\pi_{*}\left(\operatorname{Sym}^{m} H^{0}(\mathbb{P}(E), L) \otimes \mathcal{O}_{\mathbb{P}(E)}\right) \rightarrow \pi_{*}\left(L^{m}\right)$ is surjective at the point $x \in X$. As $\pi_{*}\left(L^{m}\right)=\operatorname{Sym}^{2 a b m} E \otimes H^{2 b m}$ we may conclude that for any $a>0$ and $m$ large enough the vector bundle $\operatorname{Sym}^{2 a b m} E \otimes H^{2 b m}$ is generated at $x$ by its global sections, hence $x \notin \mathbb{B}_{-}(E)$.

The analogous claim holds for augmented base locus, with a similar proof.

Proposition 3.2 Let $E$ be a vector bundle on a smooth projective variety $X$, with the same notations as in Proposition 3.1, we have 


$$
\pi\left(\mathbb{B}_{+}\left(\mathcal{O}_{\mathbb{P}(E)}(1)\right)\right)=\mathbb{B}_{+}(E) .
$$

Proof Let $H \in \operatorname{Pic}(X)$ be a sufficiently ample line bundle such that $A=$ $\mathcal{O}_{\mathbb{P}(E)}(1) \otimes \pi^{*} H$ is very ample on $\mathbb{P}(E)$. Then

$$
\mathbb{B}_{+}\left(\mathcal{O}_{\mathbb{P}(E)}(1)\right)=\bigcap_{a>0} \mathbb{B}\left(\mathcal{O}_{\mathbb{P}(E)}(a) \otimes A^{-1}\right)=\bigcap_{a>0} \bigcap_{b>0} \operatorname{Bs}\left(\mathcal{O}_{\mathbb{P}(E)}(a b) \otimes A^{-b}\right)
$$

and

$$
\mathbb{B}_{+}(E)=\bigcap_{a>0} \bigcap_{b>0} \operatorname{Bs}\left(\operatorname{Sym}^{a b} E \otimes H^{-b}\right) .
$$

In order to show that $\pi\left(\mathbb{B}_{+}\left(\mathcal{O}_{\mathbb{P}(E)}(1)\right)\right) \subseteq \mathbb{B}_{+}(E)$, observe that if $\operatorname{Sym}^{a b} E \otimes H^{-b}$ is globally generated at a point $x \in X$, then $\pi^{*} \operatorname{Sym}^{a b} E \otimes \pi^{*} H^{-b}$ is generated at all points in $\pi^{-1}(x)$, hence $\mathcal{O}_{\mathbb{P}(E)}(a b) \otimes \pi^{*} H^{-b}=\mathcal{O}_{\mathbb{P}(E)}((a+1) b) \otimes A^{-b}$ is globally generated at all points in $\pi^{-1}(x)$.

To show the other inclusion, set $U=X \backslash \pi\left(\mathbb{B}_{+}\left(\mathcal{O}_{\mathbb{P}(E)}(1)\right)\right)$ and observe that $\left(\mathcal{O}_{\mathbb{P}(E)}(a b) \otimes A^{-b}\right)$ is generated by its global sections at the points of $\pi^{-1}(U)$ for $a$ and $b$ sufficiently large. Let us consider $b>0$ a sufficiently large positive integer, $a=(b-1) k>0$ a sufficiently large multiple of $b-1$, and set $c=((a-1) b+1) /(b-1)=k b-1=a+k-1$. Finally, let $L$ be the line bundle

$$
\begin{aligned}
L & =\mathcal{O}_{\mathbb{P}(E)}(c(b-1)) \otimes \pi^{*} H^{-(b-1)}=\mathcal{O}_{\mathbb{P}(E)}((a-1) b+1) \otimes \pi^{*} H^{-(b-1)} \\
& \simeq\left(\mathcal{O}_{\mathbb{P}(E)}(a b) \otimes\left(\mathcal{O}_{\mathbb{P}(E)}(-1) \otimes \pi^{*} H^{-1}\right)^{b}\right) \otimes\left(\mathcal{O}_{\mathbb{P}(E)}(1) \otimes \pi^{*} H\right) \\
& \simeq\left(\mathcal{O}_{\mathbb{P}(E)}(a b) \otimes A^{-b}\right) \otimes A
\end{aligned}
$$

Now for $b$ and $k$ large enough $L$ is the product of the very ample line bundle $A$ with a line bundle which is generated by global sections on $\pi^{-1}(U)$, so it is very ample on the open subset $\pi^{-1}(U)$. Furthermore, we have that $\pi_{*}(L)=\operatorname{Sym}^{c(b-1)} E \otimes H^{-(b-1)}$ and so we can apply the same argument as in the proof of Proposition 3.1 to finish the proof.

\section{Positivity properties for line bundles}

We recall here how augmented and restricted base loci are involved with various positivity notions of line bundles, as well as loci defined by negative curves:

Definition 4.1 Let $L$ be a line bundle on a smooth projective variety $X$. Fix an ample line bundle $A$ and a rational number $\varepsilon>0$. We define

(4.1.1) $T_{\varepsilon}^{A}=\overline{\left\{x: \exists C \subseteq X \text { curve on } X \text { such that } x \in C, c_{1}(L) \cdot C<\varepsilon \cdot c_{1}(A) \cdot C\right\}}$ to be the non-AEA locus of $L$ with respect to $A$ and $\varepsilon$;

(4.1.2) $\mathbb{T}(L)=\bigcap_{\varepsilon>0} T_{\varepsilon}^{A}$ to be the stable non-AEA locus of $L$, and (4.1.3) $\mathbb{T}^{0}(L)=\{x: x \in C$ such that $L \cdot C<0\}$ the negative locus of $L$. 
Proposition-Definition 4.2 For a line bundle $L$ on the variety $X$ we have the following:

(4.2.1) $\mathbb{T}^{0}(L) \subseteq \mathbb{B}_{-}(L)$, the inequality can be strict, cf. [2, Remark 6.3].

(4.2.2) $\mathbb{T}(L) \subseteq \mathbb{B}_{+}(L)$.

(4.2.3) $L$ is ample iff $\mathbb{B}_{+}(L)=\emptyset$.

(4.2.4) $L$ is semiample iff $\mathbb{B}(L)=\emptyset$.

(4.2.5) $L$ is nef iff $\mathbb{B}_{-}(L)=\varnothing$ iff $\mathbb{T}^{0}(L)=\emptyset$.

(4.2.6) $L$ is big iff $\mathbb{B}_{+}(L) \neq X$.

(4.2.7) $L$ is psef (pseudo-effective) iff $\mathbb{B}_{-}(L) \neq X$.

(4.2.8) $L$ is almost nef iff $\mathbb{T}^{0}(L)$ is contained in a countable union of proper closed subsets of $X$.

(4.2.9) $L$ is AEA (almost everywhere ample) if $\mathbb{T}(L) \neq X$.

(4.2.10) $L$ is weakly positive if $\overline{\mathbb{B}_{-}(L)} \neq X$.

Proof Points (4.2.1)-(4.2.6) are well-known statements. The claims (4.2.8)-(4.2.10) are the definitions of respective notions according to $[2,14,18]$, respectively. The only statement in need of a proof is (4.2.7).

Note that a line bundle is pseudo-effective precisely if its numerical equivalence class lies in the closure of the effective cone in the real Néron-Severi group. Hence the line bundle $L$ is psef if and only if for all $m>0, L+(1 / m) A$ is effective, or, equivalently, if for all $m>0, \mathbb{B}(m L+A) \neq X$. Therefore $\mathbb{B}_{-}(L) \neq X$ as it is contained in a countable union of proper closed subsets of $X$. Conversely, if $\mathbb{B}_{-}(L) \neq$ $X$, then the class of $L$ is a limit of effective classes.

Remark 4.3 Positivity properties related to asymptotic base loci are best summarized in the form of a table.

\begin{tabular}{lllll}
\hline & $\mathbb{B}_{-}(L)$ & $\overline{\mathbb{B}_{-}(L)}$ & $\mathbb{B}(L)$ & $\mathbb{B}_{+}(L)$ \\
\hline$=\emptyset$ & nef & nef & semiample & ample \\
\hline$\neq X$ & pseudo-effective & weakly positive & effective & big \\
\hline
\end{tabular}

Remark 4.4 See Sect. 8 and in particular Remark 8.1 for more details about non-AEA loci and their relationship with the augmented base loci.

Furthermore, as we mentioned earlier, Lesieutre [13] proved that there exist line bundles which are pseudo-effective but not weakly positive. In particular, $\mathbb{B}_{-}(L)$ is not necessarily closed.

Proposition 4.5 A line bundle $L$ is almost nef if and only if it is pseudo-effective.

Proof One implication is obvious by (4.2.1). The other implication follows from [2], as if $L$ is not pseudo-effective then there exists a reduced irreducible curve $C \subseteq X$, such that $c_{1}(L) \cdot C<0$ and $C$ moves in a family covering all $X$, so $\mathbb{T}^{0}(L)$ cannot be contained in a countable union of proper (Zariski) closed subsets. 
The following theorem will be proved in Sect. 7 .

Theorem 4.6 A line bundle $L$ is big if and only if it is AEA.

A recent result of Lehmann [12] gives a characterization of the relationship between the non-AEA locus and the diminished base locus. We will use the following when describing all the relationships.

Definition 4.7 Let $X$ be a smooth projective variety over $\mathbb{C}$ and let $D$ be a pseudoeffective $\mathbb{R}$-divisor on $X$. Suppose that $\phi: Y \rightarrow X$ is a proper birational map from a smooth variety $Y$. The movable transform of $D$ on $Y$ is defined to be

$$
\phi_{\mathrm{mov}}^{-1}(D)=\phi^{*} D-\sum_{E \phi \text {-exc. }} \sigma_{E}\left(\phi^{*} D\right) \cdot E .
$$

For a pseudo-effective line bundle $L$ define $\phi_{\text {mov }}^{-1}(L)$ as the line bundle associated to $\phi_{\text {mov }}^{-1}\left(c_{1}(L)\right)$ on $Y$.

Note that the movable transform is not linear and is only defined for pseudo-effective divisors.

Remark 4.8 In the above, $\sigma_{E}$ is the asymptotic multiplicity function introduced by Nakayama [16, Section III.1]. If $X$ is a smooth projective variety, $L$ a pseudo-effective $\mathbb{R}$-divisor, $E$ a prime divisor on $X$, then

$$
\sigma_{E}(L)=\lim _{\epsilon \rightarrow 0^{+}} \inf \left\{\operatorname{mult}_{E} L^{\prime}: L^{\prime} \geqslant 0 \text { and } L^{\prime} \sim_{\mathbb{R}} L+\epsilon A\right\}
$$

where $A$ is an arbitrary but fixed ample divisor.

Remark 4.9 Following [12, Definition 1.2], we call an irreducible curve $C$ on $X$ to be a mov ${ }^{1}$-curve, if it deforms to cover a codimension one subset of $X$.

Theorem 4.10 ([12]) Let $X$ be a smooth projective variety over $\mathbb{C}$ and $D$ a pseudoeffective $\mathbb{R}$-divisor. $D$ is not movable if and only if there is a mov ${ }^{1}$-curve $C$ on $X$ and a proper birational morphism $\phi: Y \rightarrow X$ from a smooth variety $Y$ such that

$$
\phi_{\text {mov }}^{-1}(D) \cdot \bar{C}<0,
$$

where $\bar{C}$ is the strict transform of a generic deformation of $C$.

The following reformulation is easy to see.

Theorem 4.11 Let $X$ be a smooth projective variety and $D$ a pseudo-effective $\mathbb{R}$ divisor. Suppose that $V$ is an irreducible subvariety of $X$ contained in $\mathbb{B}_{-}(L)$ and let $\psi: X^{\prime} \rightarrow X$ be a smooth birational model resolving the ideal sheaf of $V$. Then there is a birational morphism $\phi: Y \rightarrow X^{\prime}$ from a smooth variety $Y$ and an irreducible curve $\bar{C}$ on $Y$ such that $\phi_{\mathrm{mov}}^{-1}\left(\psi^{*} D\right) \cdot \bar{C}<0$ and $\psi \circ \varphi(\bar{C})$ deforms to cover $V$. 
Remark 4.12 Let $L$ be any line bundle on $X$ smooth projective, then

$$
\mathbb{B}_{-}(L)=\bigcup_{f: Y \rightarrow X} f\left(\mathbb{T}^{0}\left(f_{\text {mov }}^{-1}(L)\right)\right)
$$

There are several examples for which the loci $\mathbb{T}^{0}(L)$ and $\mathbb{B}_{-}(L)$ do not coincide, and in some cases the difference is divisorial.

Question 4.13 Is it true that $\mathbb{B}_{-}(L)$ is contained in a proper closed subset of $X$ (i.e., $L$ is weakly positive) if and only if the same holds for $\mathbb{T}^{0}(L)$ ?

Remark 4.14 In [2, Question 7.5] the authors ask if for a vector bundle $E$, $\mathbb{B}_{-}\left(\mathcal{O}_{\mathbb{P}(E)}(1)\right)$ does not dominate $X$ if and only if neither does $\mathbb{T}^{0}\left(\mathcal{O}_{\mathbb{P}(E)}(1)\right)$.

\section{Restricted base loci for vector bundles}

Here we explore the connections between the positivity properties of a vector bundle and the associated asymptotic base loci. We will start recalling some classical definitions for vector bundles. Note that these definitions do sometimes appear slightly differently in the literature, but we will try to follow and indicate specific selected references each time.

Definition 5.1 Let $E$ be a vector bundle on a smooth projective variety $X, \pi: \mathbb{P}(E) \rightarrow$ $X$ the projective bundle of one dimensional quotients of $E$, and $\mathcal{O}_{\mathbb{P}(E)}(1)$ the universal quotient of $\pi^{*} E$ on $\mathbb{P}(E)$. We say that $E$ is

(5.1.1) nef if $\left.\mathcal{O}_{\mathbb{P}(E)}(1)\right)$ is a nef line bundle, i.e., if $\mathbb{B}_{-}\left(\mathcal{O}_{\mathbb{P}(E)}(1)\right)=\emptyset$;

(5.1.2) almost nef if $\pi\left(\mathbb{T}^{0}\left(\mathcal{O}_{\mathbb{P}(E)}(1)\right)\right)$ is contained in a countable union of proper closed subsets of $X$, cf. [2];

(5.1.3) pseudo-effective if $\mathbb{B}_{-}(E) \neq X$, cf. [2];

(5.1.4) weakly positive if $\overline{\mathbb{B}_{-}(E)} \neq X$, cf. [18].

From Proposition 3.1 we have immediately the following two statements.

Proposition 5.2 A vector bundle $E$ is nef if and only if $\mathbb{B}_{-}(E)=\emptyset$.

Proposition 5.3 A vector bundle $E$ is pseudo-effective if and only if $\mathcal{O}_{\mathbb{P}(E)}(1)$ is pseudo-effective and $\pi\left(\mathbb{B}_{-}\left(\mathcal{O}_{\mathbb{P}(E)}(1)\right)\right) \neq X$.

Remark 5.4 The proposition above is the same as [2, Proposition 7.2]. Observe that the locus $L_{\text {nonnef }}$ in [2] is what we call $\mathbb{B}_{-}(L)$ here (we are in the smooth projective case).

The following proposition is immediate.

Proposition 5.5 A vector bundle $E$ is almost nef if and only if there exists a countable union $T=\bigcup_{i \in \mathbb{N}} T_{i}$ of proper closed subsets of $X$, such that for any curve $C \subseteq X$ not contained in $T$ the restriction $\left.E\right|_{C}$ is a nef vector bundle. 
Remark 5.6 It follows from the definitions and propositions above that

$$
E \text { weakly positive } \Longrightarrow E \text { pseudo-effective } \Longrightarrow E \text { almost nef. }
$$

We have seen that the first implication is not an equivalence, while it is an open question whether, for vector bundles, being almost nef is equivalent to being pseudo-effective, as in the line bundle case, cf. [2, Question 7.5].

Question 5.7 Does $E$ being almost nef imply that $E$ is pseudo-effective?

If $E$ is almost nef, then the line bundle $\mathcal{O}_{\mathbb{P}(E)}(1)$ is almost nef, hence pseudo-effective. In order to have that $E$ is pseudo-effective, one needs to show that $\mathbb{B}_{-}\left(\mathcal{O}_{\mathbb{P}(E)}(1)\right)$ does not dominate $X$.

\section{Augmented base loci for vector bundles}

Definition 6.1 Let $E$ be a vector bundle on a smooth projective variety $X, \pi: \mathbb{P}(E) \rightarrow$ $X$ the projective bundle of one dimensional quotients of $E$, and $\mathcal{O}_{\mathbb{P}(E)}(1)$ the universal quotient of $\pi^{*} E$ on $\mathbb{P}(E)$. We say that

(6.1.1) $E$ is ample if $\mathcal{O}_{\mathbb{P}(E)}(1)$ is ample on $\mathbb{P}(E)$;

(6.1.2) $E$ is $L$-big if $\mathcal{O}_{\mathbb{P}(E)}(1)$ is big on $\mathbb{P}(E)$;

(6.1.3) $E$ is V-big (or Viehweg-big) if there exists an ample line bundle $A$ and a positive integer $c>0$ such that $\operatorname{Sym}^{c} E \otimes A^{-1}$ is weakly positive, i.e., such that $\overline{\mathbb{B}_{-}\left(\operatorname{Sym}^{q} E \otimes A^{-1}\right)} \subsetneq X$, cf. (5.1.4).

Directly from Proposition 3.2 we have

Proposition 6.2 A vector bundle $E$ is ample if and only if $\mathbb{B}_{+}(E)=\emptyset$.

Remark 6.3 It is well-known that a line bundle $L$ is big if and only if $\mathbb{B}_{+}(L) \neq X$, equivalently, if there exist $A$ ample and a positive integer $c>0$ such that $L^{\otimes c} \otimes A^{-1}$ is pseudo-effective. Next we will prove that the same equivalences hold for V-bigness for vector bundles of arbitrary rank.

Theorem 6.4 Let $E$ be a vector bundle on a smooth projective variety $X$. Then the following properties are equivalent:

(6.4.1) E is V-big.

(6.4.2) There exist an ample line bundle $A$ and a positive integer $c>0$ such that $\mathrm{Sym}^{c} E \otimes A^{-1}$ is pseudo-effective.

(6.4.3) $\mathbb{B}_{+}(E) \neq X$.

Proof The implication $(6.4 .1) \Rightarrow(6.4 .2)$ is clear; let us consider (6.4.2) $\Rightarrow(6.4 .3)$. Suppose there exist $A$ ample and $c>0$ such that $\operatorname{Sym}^{c} E \otimes A^{-1}$ is pseudo-effective, i.e.,

$$
\mathbb{B}_{-}\left(\operatorname{Sym}^{c} E \otimes A^{-1}\right) \neq X
$$


Then

$$
\bigcup_{q>0} \mathbb{B}\left(E-\frac{1}{c} A+\frac{1}{q} A\right)=\bigcup_{q>0} \mathbb{B}\left(E-\frac{q-c}{q c} A\right) \neq X .
$$

Now for $q \gg 0$ and $(q-c) /(q c)>1 /(2 c)$, one has

$$
\mathbb{B}_{+}(E) \subseteq \mathbb{B}\left(E-\frac{1}{2 c} A\right) \subseteq \mathbb{B}\left(E-\frac{q-c}{q c} A\right) \subsetneq X
$$

hence the validity of the desired implication.

Next we verify that $(6.4 .3) \Rightarrow(6.4 .1)$. If $E$ satisfies $\mathbb{B}_{+}(E) \neq X$, then there exists $q>0$ such that $\mathbb{B}(E-(1 / q) A) \subsetneq X$ is a closed proper subset. Consequently,

$$
\overline{\mathbb{B}_{-}\left(\operatorname{Sym}^{q} E \otimes A^{-1}\right)} \subseteq \mathbb{B}\left(\operatorname{Sym}^{q} E \otimes A^{-1}\right) \subsetneq X
$$

Corollary 6.5 If E is V-big, then it is L-big as well.

Proof Theorem 6.4 yields $\mathbb{B}_{+}(E) \neq X$, therefore $\mathbb{B}_{+}\left(\mathcal{O}_{\mathbb{P}(E)}(1)\right) \neq \mathbb{P}(E)$ via Proposition 3.2. As a consequence $\mathcal{O}_{\mathbb{P}(E)}(1)$ is big on $\mathbb{P}(E)$, equivalently, $E$ is L-big.

Remark 6.6 L-big vector bundles are not necessarily V-big, as the example of $\mathcal{O}_{\mathbb{P}^{1}} \oplus \mathcal{O}_{\mathbb{P}^{1}}(1)$ on $\mathbb{P}^{1}$ shows, see [8, p. 24].

The key difference between V-big and L-big vector bundles is that being L-big means that $\mathcal{O}_{\mathbb{P}(E)}(1)$ is ample with respect to an open set $V \subseteq \mathbb{P}$ whereas $E$ is V-big if we can take $V$ to be of the form $V=\pi^{-1}(U), U \subseteq X$ open.

Remark 6.7 A V-big vector bundle $E$ on a variety $X$ is also called ample with respect to an open subset, cf. [8, Chapter 3] and [9]. A similar notion to V-bigness, strong bigness, has been defined by Brotbek for vector bundles, cf. [3].

Remark 6.8 In the case where $E=\Omega_{X}$ is the cotangent sheaf of a variety $X$, the definitions and Proposition 3.2 imply the following inclusion $\mathbb{B}_{+}\left(\Omega_{X}\right) \supseteq \operatorname{DS}\left(X, T_{X}\right)$, where $\operatorname{DS}\left(X, T_{X}\right)$ is the Demailly-Semple locus.

The work of Diverio and Rousseau [4] therefore provides examples of complex projective varieties of general type $X$ where $\Omega_{X}$ is a semistable L-big vector bundle with a big determinant, which is nevertheless not V-big.

\section{Almost everywhere ampleness}

The notion of almost everywhere ampleness was first defined by Miyaoka in the context of his work on vector bundles on surfaces; the definition goes through in all dimensions verbatim. 
Definition 7.1 ([14]) Let $X$ be a smooth projective variety, $E$ a rank $r$ vector bundle on $X$. Consider the projectivized bundle $\mathbb{P}=\mathbb{P}(E)$ with projection morphism $\pi: \mathbb{P} \rightarrow X$ and tautological bundle $\mathcal{O}_{\mathbb{P}}(1)$. We say that $E$ is almost everywhere ample (AEA for short), if there exists an ample line bundle $A$ on $X$, a Zariski closed subset $T \subset \mathbb{P}$, whose projection $\pi(T)$ onto $X$ satisfies $\pi(T) \neq X$, and a positive number $\varepsilon>0$ such that

$$
c_{1}\left(\mathcal{O}_{\mathbb{P}}(1)\right) \cdot C \geqslant \varepsilon \cdot \pi^{*}\left(c_{1}(A)\right) \cdot C
$$

for all curves $C \subset \mathbb{P}$ that are not contained in $T$.

For line bundles, this notion coincides with bigness.

Proposition 7.2 For a line bundle $L$ on a smooth projective variety $X$, the following are equivalent:

(7.2.1) L is AEA, i.e, there is an ample line bundle $A$ on $X$, a number $\varepsilon>0$, and a proper Zariski closed subset $T \subset X$ such that

$$
c_{1}(L) \cdot C \geqslant \varepsilon \cdot c_{1}(A) \cdot C
$$

for all curves $C \subset X$ not contained in $T$.

(7.2.2) For every ample line bundle $A$ on $X$, there is $\varepsilon>0$ and a proper Zariski closed subset $T \subset X$ such that

$$
c_{1}(L) \cdot C \geqslant \varepsilon \cdot c_{1}(A) \cdot C
$$

for all curves $C \subset X$ not contained in $T$.

(7.2.3) L is big.

Proof Assume (7.2.3), and let $A$ be any ample line bundle. Then, by Kodaira's lemma, there is a positive integer $m$ such that we can write

$$
m c_{1}(L)=c_{1}(A)+F,
$$

where $F$ is an effective divisor. Taking $T$ to be the support of $F$, it follows for every curve $C \subset X$ not contained in $T$ that

$$
m c_{1}(L) \cdot C=c_{1}(A) \cdot C+F \cdot C \geqslant c_{1}(A) \cdot C
$$

and this implies (7.2.2) with $\varepsilon=1 / \mathrm{m}$.

Obviously (7.2.2) implies (7.2.1), so let us assume condition (7.2.1) and show that it implies (7.2.3). A curve $C \subset X$ such that $c_{1}(L) \cdot C<\varepsilon \cdot c_{1}(A) \cdot C$ cannot be a movable curve (in the sense of [11, Section 11.4.C]), since these cover all of $X$ (by [11, Lemma 11.4.18]), whereas $T \neq X$. So $L$ must have positive intersection with all movable curves. This implies that $L$ lies in the dual of the cone of movable curves $\operatorname{Mov}(X)$, which by the theorem of Boucksom-Demailly-Păun-Peternell [2] is the 
pseudo-effective cone $\overline{\mathrm{Eff}}(X)$. In order to conclude that $L$ is big-and thus to complete the proof-it is therefore enough to show that $L$ lies in the interior of that cone.

The assumption that $L$ is AEA says that

$$
\left(c_{1}(L)-\varepsilon c_{1}(A)\right) C \geqslant 0 \text { for all } C \not \subset T .
$$

Therefore, writing $c_{1}(L)-\varepsilon c_{1}(A)=\left(c_{1}(L)-\varepsilon c_{1}(A) / 2\right)-\varepsilon c_{1}(A) / 2$, we see that $c_{1}(L)-\varepsilon c_{1}(A) / 2$ is AEA as well. Moreover, every class in the open set

$$
c_{1}(L)-\frac{\varepsilon}{2} c_{1}(A)+\operatorname{Amp}(X)
$$

is AEA, and $c_{1}(L)$ lies in this open set.

Proposition 7.3 Let $E$ be a vector bundle on a smooth projective variety $X$, let $\mathbb{P}=$ $\mathbb{P}(E)$. If $E$ is $A E A$ on $X$, then so is $\mathcal{O}_{\mathbb{P}}(1)$ on $\mathbb{P}$.

Proof For $E$ to be AEA means that for every ample line bundle $A$ on $X$, there exists a Zariski-closed subset $T \subseteq \mathbb{P}$ not dominating $X$, and $\varepsilon>0$ such that

$$
c_{1}\left(\mathcal{O}_{\mathbb{P}}(1)\right) \cdot C \geqslant \varepsilon\left(\pi^{*} c_{1}(A) \cdot C\right)
$$

for all irreducible curves not contained in $T$.

Since $\mathcal{O}_{\mathbb{P}}(1)$ is $\pi$-ample, the line bundle $\pi^{*} A^{m} \otimes \mathcal{O}_{\mathbb{P}}(1)$ is ample for all $m \geqslant$ $m_{0} \gg 0$ by [11, Proposition 1.7.10]. Let us choose $m_{0}$ a positive integer such that $\pi^{*} A^{m_{0}} \otimes \mathcal{O}_{\mathbb{P}}(1)$ is ample.

We will prove that $\mathcal{O}_{\mathbb{P}}(1)$ is AEA on $\mathbb{P}$ with closed subset $T \nsubseteq \mathbb{P}$, ample line bundle $\pi^{*} A^{m_{0}} \otimes \mathcal{O}_{\mathbb{P}}(1)$ on $\mathbb{P}$, and a suitable $\varepsilon^{\prime}>0$. We need that

$$
c_{1}\left(\mathcal{O}_{\mathbb{P}}(1)\right) \cdot C \geqslant \varepsilon^{\prime}\left(c_{1}\left(\pi^{*} A^{m_{0}} \otimes \mathcal{O}_{\mathbb{P}}(1)\right) \cdot C\right),
$$

or equivalently,

$$
c_{1}\left(\mathcal{O}_{\mathbb{P}}(1)\right) \cdot C \geqslant \frac{m_{0} \varepsilon^{\prime}}{1-\varepsilon^{\prime}}\left(\pi^{*} c_{1}(A) \cdot C\right)
$$

for all curves not contained in $T$. By our assumption on $E$, this holds whenever

$$
\varepsilon^{\prime}<\frac{\varepsilon}{\varepsilon+m_{0}}
$$

Corollary 7.4 If E is AEA, then it is L-big.

Proof Immediately from Propositions 7.2 and 7.3 


\section{The bad AEA locus in the line bundle case}

Throughout this section we will prove some relationships between the loci $\mathbb{T}(L), \mathbb{B}_{-}(L), \mathbb{B}_{+}(L)$, and provide some examples.

Consider a line bundle $L$, and fix an ample line bundle $A$ and a number $\varepsilon>0$. We defined the non-AEA locus of $L$ with respect to $A$ and $\varepsilon$ as the subvariety

$$
T_{\varepsilon}^{A}=\overline{\bigcup\left\{C: C \text { curve on } X \text { with } c_{1}(L) \cdot C<\varepsilon \cdot c_{1}(A) \cdot C\right\}} .
$$

The AEA assumption on $L$ simply means that there exists $\varepsilon>0$ such that $T_{\varepsilon}^{A} \neq X$. For $\varepsilon<\delta$ we have $T_{\varepsilon} \subset T_{\delta}$, so that we can express the AEA condition equivalently as saying that the intersection $\mathbb{T}(L)=\bigcap_{\varepsilon>0} T_{\varepsilon}^{A}$ is not all of $X$.

Remark 8.1 It is immediate that $\mathbb{T}(L) \subseteq \mathbb{B}_{+}(L)$. In fact, by the noetherian property there are positive real numbers $\varepsilon_{0}$ and $\delta_{0}$ such that

$$
\begin{aligned}
\mathbb{T}(L) & =T_{\varepsilon}^{A} & & \text { for all } \varepsilon \leqslant \varepsilon_{0}, \\
\mathbb{B}_{+}(L) & =\mathbb{B}(L-\delta A) & & \text { for all } \delta \leqslant \delta_{0} .
\end{aligned}
$$

Now choose $\varepsilon<\min \left(\varepsilon_{0}, \delta_{0}\right)$, then

$$
\mathbb{T}(L)=T_{\varepsilon}=\overline{\left\{x: x \in C \text { curve on } X \text { such that } c_{1}(L) \cdot C<\varepsilon \cdot c_{1}(A) \cdot C\right\}} .
$$

If $C$ is a curve such that $(L-\varepsilon A) C<0$ then $C \subseteq \mathbb{B}(L-\varepsilon A)=\mathbb{B}_{+}(L)$ which is a closed set.

Remark 8.2 A line bundle $L$ is ample if and only if $\mathbb{T}(L)=\emptyset$. In general the inclusion

$$
\overline{\left\{x: x \in C \text { curve on } X \text { such that } c_{1}(L) \cdot s C \leqslant 0\right\}} \subseteq \mathbb{T}(L)
$$

is strict, as shown by a strictly nef non ample line bundle $L$, where the first set is empty but the second one is not. Examples of line bundles that are strictly nef (and even big) and non ample have been first given by Mumford, cf. [7], and a complete description can be found in [17].

Remark 8.3 A few words on the relationship between $\mathbb{T}(L)$ and $\mathbb{B}_{ \pm}(L)$. We will show here that $\mathbb{T}(L) \neq \mathbb{B}_{+}(L)$ in general. A bit more precisely, we will try to understand the relationship of $\mathbb{T}(L)$ to the augmented and restricted base loci of $L$ when $\operatorname{dim} X=2$. Recall that

$$
\mathbb{B}_{-}(L)=\bigcup_{m=1}^{\infty} \mathbb{B}\left(L+\frac{1}{m} A\right)
$$

for any integral ample divisor $A$ on $X$. 
Let $D$ be a big divisor on a smooth projective surface $X$ with Zariski decomposition $D=P_{D}+N_{D}$. Then [5, Examples 1.11 and 1.17] tell us that

$$
\begin{aligned}
& \mathbb{B}_{+}\left(\mathcal{O}_{X}(D)\right)=\operatorname{Null}\left(P_{D}\right)=\bigcup_{C}\left\{C \subseteq X \text { irred }: P_{D} \cdot C=0\right\}, \\
& \mathbb{B}_{-}\left(\mathcal{O}_{X}(D)\right)=\operatorname{Supp} N_{D} .
\end{aligned}
$$

Example 8.4 Here we present an example where $\mathbb{T}(L) \neq \mathbb{B}_{ \pm}(L)$. Let $X$ be a surface that carries a big divisor $D$ and an irreducible curve $C \subseteq X$ satisfying $C \subseteq \operatorname{Supp} N_{D}$ and $D \cdot C>0$. Then $C \nsubseteq \mathbb{T}\left(\mathcal{O}_{X}(D)\right)$, but $C \subseteq \mathbb{B}_{-}\left(\mathcal{O}_{X}(D)\right) \subseteq \mathbb{B}_{+}\left(\mathcal{O}_{X}(D)\right)$. In this case we have

$$
\mathbb{T}\left(\mathcal{O}_{X}(D)\right) \neq \mathbb{B}_{-}\left(\mathcal{O}_{X}(D)\right), \mathbb{B}_{+}\left(\mathcal{O}_{X}(D)\right)
$$

Surfaces carrying such $D$ and $C$ exist by [1]: Consider a K3 surface $X$, on which the Zariski chamber decomposition does not coincide with the Weyl chamber decomposition. The latter is by [1, Theorem 1] the case if and only if there are (-2)-curves on $X$ having intersection number 1 . For a concrete example one can, as done in [1, Section 3], take a smooth quartic surface $X \subset \mathbb{P}^{3}$ that has a hyperplane section of the form $H=L_{1}+L_{2}+Q$, where $L_{1}$ and $L_{2}$ are lines and $Q$ is a smooth conic. Then the divisors of the form

$$
D=H+a_{1} L_{1}+a_{2} L_{2}
$$

with $a_{1} \geqslant 1$ and $a_{2} \geqslant 1$ have $L_{1}$ and $L_{2}$ in the support of the negative part of their Zariski decomposition, but one can find $a_{1}, a_{2}$ such that $D \cdot L_{1}>0$ and $D \cdot L_{2}<0$ (for instance $a_{1}=2, a_{2}=4$ ). (In the notation of [1], $D$ lies in the Zariski chamber $Z_{\left\{L_{1}, L_{2}\right\}}$, but not in the Weyl chamber $W_{\left\{L_{1}, L_{2}\right\}}$, instead it lies in the Weyl chamber $W_{\left\{L_{2}\right\}}$.)

Example 8.5 In general $\mathbb{T}\left(\mathcal{O}_{X}(D)\right)$ is not contained in $\mathbb{B}_{-}\left(\mathcal{O}_{X}(D)\right)$ either, where $L=\mathcal{O}_{X}(D)$ for a suitable integral Cartier divisor $D$. To see this, take a surface where all negative curves have self-intersection -1 . Then the intersection form of the negative part of the Zariski decomposition of any big divisor is - Id, in other words, no two curves in it can intersect. Consequently,

$$
\mathbb{T}\left(\mathcal{O}_{X}(D)\right)=\mathbb{B}_{+}\left(\mathcal{O}_{X}(D)\right)
$$

This can be seen as follows: let $C \subseteq \mathbb{B}_{+}\left(\mathcal{O}_{X}(D)\right)=\operatorname{Null}\left(P_{D}\right)$ be an irreducible curve. Since $P_{D}$ is big and nef, the intersection form on $P_{D}^{\perp}$ is negative definite, which under the given circumstances means that $\left(C \cdot C^{\prime}\right)=0$ for every irreducible curve $C \neq C^{\prime}$ coming up in $N_{D}$. Therefore

$$
D \cdot C=P_{D} \cdot C+N_{D} \cdot C=0+(\leqslant 0)=(\leqslant 0) .
$$

Consequently, $C \subseteq \mathbb{T}\left(\mathcal{O}_{X}(D)\right)$.

Take a non-stable (in the sense of [5, Definition 1.22]) big divisor $D$ on $X$, then $\mathbb{B}_{-}\left(\mathcal{O}_{X}(D)\right) \subsetneq \mathbb{B}_{+}\left(\mathcal{O}_{X}(D)\right)=\mathbb{T}\left(\mathcal{O}_{X}(D)\right)$. 


\section{V-big versus AEA}

Let $X$ be a smooth projective variety and $E$ a vector bundle on $X$. There exist two non-equivalent definitions for bigness in the literature: V-big and L-big vector bundles. It is known that V-bigness implies L-bigness and that the converse does not hold if rk $E \geqslant 2$, cf. Remark 6.8 .

Throughout this section we will point out some differences (for example a different Kodaira's lemma) between L-big and V-big vector bundles, and compare V-bigness and almost everywhere ampleness. In particular we will show that these positivity properties coincide for nef vector bundles.

Remark 9.1 In the literature V-big vector bundles are also called ample with respect to an open set $U[9]$, with $U \subseteq \mathbb{B}_{+}(E)$. We will refer to them in such way when stating properties proven in [8], or when we want to stress the emphasis on the open sets involved.

We have seen that if $E$ is V-big, then $E$ is also AEA: apply Remark 8.1 to $\mathbb{T}\left(\mathcal{O}_{\mathbb{P}(E)}(1)\right)$.

Question 9.2 Does $E$ being AEA imply that $E$ is V-big? We will see that this is the case if $E$ is nef.

Remark 9.3 As pointed out in [8, Lemma 3.44], a vector bundle on a projective curve is ample with respect to an open set (i.e. V-big) exactly if it is ample.

Next we will show that a strong form of Kodaira's lemma is valid for vector bundles that are ample with respect to an open set.

Lemma 9.4 (Kodaira's lemma for vector bundles) Let $X$ be a smooth projective variety, $E$ a vector bundle, and $A$ an ample line bundle on $X$. Then the following are equivalent:

(9.4.1) E is ample with respect to an open subset.

(9.4.2) $\mathrm{Sym}^{m} E$ contains an ample vector bundle of the same rank for some $m>0$.

(9.4.3) There exists $m>0$ and an injective morphism

$$
\bigoplus^{\mathrm{rk} \operatorname{Sym}^{m} E} A \hookrightarrow \operatorname{Sym}^{m} E
$$

which is an isomorphism over an open subset.

Proof The equivalence of (9.4.1) and (9.4.3) is the content of [8, Lemma 3.42]; (9.4.3) obviously implies (9.4.2), and (9.4.2) implies (9.4.1) holds since being ample with respect to an open set is scale-invariant.

There is a useful characterization of ampleness with respect to an open subset in terms of $\mathcal{O}_{\mathbb{P}(E)}(1)$.

Lemma 9.5 (cf. Proposition 3.2) With notation as above, $E$ is ample with respect to the dense open set $U \subseteq X$ precisely if $\mathcal{O}_{\mathbb{P}(E)}(1)$ is ample with respect to $\pi^{-1}(U) \subseteq \mathbb{P}(E)$.

Here we have the following weaker version of the Kodaira lemma. 
Lemma 9.6 Let $E$ be a vector bundle using the notation above.

(9.6.1) Assume that $H^{0}\left(X, \operatorname{Sym}^{m} E\right) \neq 0$ for some $m>0$. Then for any ample line bundle $A$ on $X$ and any $k>0, \operatorname{Sym}^{k} E \otimes A$ is L-big.

(9.6.2) Assume that for some $m>0$ and some $x \in X$ the vector bundle $\mathrm{Sym}^{m} E$ is generated at $x$ by its global sections $H^{0}\left(X, \operatorname{Sym}^{m} E\right)$. Then for any ample line bundle $A$ on $X$ and any $k>0, \operatorname{Sym}^{k} E \otimes A$ is $V$-big.

(9.6.3) Conversely, assume that $E$ is L-big. Then for any line bundle $L$ on $X$,

$$
H^{0}\left(X, \operatorname{Sym}^{m} E \otimes L\right) \neq 0
$$

for all $m \gg 0$.

(9.6.4) Assume that $E$ is V-big. Then for any line bundle $L$ on $X, \mathrm{Sym}^{m} E \otimes L i s$ generically generated by its global sections for all $m \gg 0$.

Proof To prove (9.6.1), assume that $H^{0}\left(X, \operatorname{Sym}^{m} E\right) \neq 0$ for some $m>0$. This means that $H^{0}\left(\mathbb{P}(E), \mathcal{O}_{\mathbb{P}(E)}(m)\right) \simeq H^{0}\left(X, \operatorname{Sym}^{m} E\right) \neq 0$, hence $\mathcal{O}_{\mathbb{P}(E)}(1)$ is $\mathbb{Q}$-effective. Then

$$
\mathcal{O}_{\mathbb{P}(E \otimes A)}(1) \simeq \mathcal{O}_{\mathbb{P}(E)}(1) \otimes \pi^{*} A
$$

By [11, Proposition 1.7.10], the $\mathbb{Q}$-divisor $a c_{1}\left(\mathcal{O}_{\mathbb{P}(E)}(1)\right)+\pi^{*} c_{1}(A)$ is ample for $0<a \ll 1$. This implies that

$$
c_{1}\left(\mathcal{O}_{\mathbb{P}(E)}(1)\right)+\pi^{*} c_{1}(A)=(1-a) c_{1}\left(\mathcal{O}_{\mathbb{P}(E)}(1)\right)+\left(a c_{1}\left(\mathcal{O}_{\mathbb{P}(E)}(1)\right)+\pi^{*} c_{1}(A)\right)
$$

can be written as the sum of an effective and an ample divisor, hence it is big.

To prove (9.6.2), assume that for some $m>0$ and some $x \in X$ the vector bundle $\operatorname{Sym}^{m} E$ is generated at $x$ by its global sections. Then $\mathbb{B}(E) \neq X$ and hence $\mathbb{B}_{-}(E) \subset$ $\mathbb{B}(E) \neq X$. Therefore $E$ is weakly positive and for all $H$ ample

$$
\mathbb{B}_{+}\left(E+\frac{1}{m} H\right)=\bigcap \mathbb{B}\left(E+\frac{1}{m} H-\frac{1}{n} H\right) \subseteq \mathbb{B}(E) \neq X .
$$

(9.6.3) is a reformulation of the Kodaira lemma on $\mathbb{P}(E)$, see [11, Lemma 2.2.6].

Finally, for (9.6.4), assume that $E$ is V-big, $H$ an ample line bundle, and $L$ any line bundle on $X$. We have that for $m$ sufficiently large $H-(1 / m) L$ is ample, and as $E$ is V-big we have

$$
\mathbb{B}\left(\operatorname{Sym}^{m k} E+L\right)=\mathbb{B}\left(\operatorname{Sym}^{k} E+\frac{1}{m} L\right) \subseteq \mathbb{B}\left(\operatorname{Sym}^{k} E-\left(H-\frac{1}{m} L\right)\right) \neq X
$$

for $k$ big enough.

Remark 9.7 A line bundle is (V-)big if and only if it is ample with respect to an open set. We have seen in Remark 6.6 that there exist simple vector bundles that are L-big, but not V-big, and hence not ample with respect to an open set. 
The following proposition allows to identify the open sets involved in almost everywhere ampleness and ampleness with respect to an open subset.

Proposition 9.8 Let $X$ be a smooth projective variety, $E$ a vector bundle on $X$ containing an ample vector bundle A of the same rank. Then

(9.8.1) $E$ is almost everywhere ample with respect to the closed subset $T=$ $\operatorname{Supp}(E / A)$.

(9.8.2) E is ample with respect to the dense open set $X \backslash T$.

Proof Let $H$ be an ample line bundle on $X$, and $L=\mathcal{O}_{\mathbb{P}(E)}(1)$ and $C \subseteq \mathbb{P}(E)$ an irreducible curve. If $C$ is contained in a fibre that maps to a point away from $T$, then

$$
L \cdot C=\pi^{*} A \cdot C
$$

which verifies the intersection condition for almost everywhere ampleness. If $C$ is contained in a fibre mapping to $T$, then we do not care about the intersections numbers at all.

We may now assume that $C$ is not contained in a fibre of $\pi$. Let $B=\pi(C) \subseteq X$. By restricting everything to $B$ via base change along $B \hookrightarrow X$, and passing to the normalization of $B$, we may assume that $X$ is a curve, and $\left.\pi\right|_{C}: C \rightarrow X$ is a dominant morphism. Consider the short exact sequence of sheaves

$$
0 \rightarrow A \rightarrow E \rightarrow Q \rightarrow 0
$$

where $Q=E / A$ is a torsion sheaf on $X$ with support $T$.

The vector bundle map $\pi^{*} E \rightarrow \mathcal{O}_{\mathbb{P}(E)}(1)$ is surjective, hence $\pi^{*} A$ maps surjectively onto a sub-line-bundle $\mathcal{B}$ of $\mathcal{O}_{\mathbb{P}(E)}(1)$. Since $\pi^{*} A$ is ample, so is the quotient B.

Remark 9.9 In the case of a line bundle $L$, the largest open subset over which the evaluation map

$$
H^{0}(X, L) \otimes \mathcal{O}_{X} \rightarrow L
$$

is surjective is the complement of the stable base locus $X \backslash \mathbb{B}(L)$.

An L-big vector bundle $E$ is V-big if $\mathbb{B}_{+}\left(\mathcal{O}_{\mathbb{P}(E)}(1)\right)$ is contained in a union of fibres over a proper Zariski closed subset of $X$.

When $L$ is a line bundle on a surface, then it is immediate from the intersectiontheoretic characterizations that

$$
\mathbb{B}_{+}(L)=\mathbb{T}\left(P_{D}\right)
$$

Since we would need something along these lines on $\mathbb{P}(E)$, which in interesting cases has dimension at least three, the above observations can only serve as a pointer what kind of statements we would like to prove in higher dimensions.

Lemma 9.10 Let $X$ be an irreducible projective variety, L a nef line bundle on $X$. Then 


$$
\mathbb{B}_{+}(L)=\mathbb{T}(L)
$$

Proof We have already observed in Remark 8.1 that $\mathbb{T}(L) \subseteq \mathbb{B}_{+}(L)$ in general. To prove the other direction in the case where $L$ is nef, we use the main result of [15], see also [6, Corollary 5.6]:

$$
\mathbb{B}_{+}(L)=\bigcup_{\substack{V \subseteq X \\ L^{d} \cdot V=0}} V .
$$

We have to show that if $V \subseteq X$ is an irreducible subvariety for which $\left.L\right|_{V}$ is not big, then $V$ is contained in $\mathbb{T}(L)$. Let us fix an ample divisor $A$ on $X$. We will show that $V$ is covered by curves $C$ satisfying $L \cdot C<\varepsilon A \cdot C$ for $\varepsilon$ small enough.

Let $\mu: V^{\prime} \rightarrow V \subseteq X$ be a resolution of singularities. Since $\left.L\right|_{V}$ was pseudoeffective and not big to begin with, then the same applies to $\mu^{*}\left(\left.L\right|_{V}\right)$. As $\mu^{*}\left(\left.L\right|_{V}\right)$ is not big, it is not in the interior of the pseudo-effective cone, hence there must exist a real 1-cycle $0 \neq C \in N_{1}\left(V^{\prime}\right)$ that is a limit of moving curves $C_{n} \subseteq V$ with $\mu^{*}\left(\left.L\right|_{V}\right) \cdot C=0$.

Now let $A$ be an ample divisor on $X$, then $\mu^{*}(A)$ is big on $V^{\prime}$, so $\mu^{*}(A) \cdot C>0$. Then

$$
\lim \frac{\mu^{*}\left(\left.L\right|_{V}\right) \cdot C_{n}}{\mu^{*}(A) \cdot C_{n}}=0 .
$$

Thus, for $n$ sufficiently large, $\mu^{*}\left(\left.L\right|_{V}\right) \cdot C_{n}<\varepsilon \mu^{*}(A) \cdot C_{n}$, hence by projection formula $L \cdot \mu_{*}\left(C_{n}\right)<\varepsilon A \cdot \mu_{*}\left(C_{n}\right)$. And the class of $\mu_{*}\left(C_{n}\right)$ covers $V$, which implies $V \subseteq \mathbb{T}(L)$.

Proposition 9.11 Let $E$ be a nef vector bundle on an irreducible projective variety $X$. Then $E$ is AEA if and only if it is V-big.

Proof Both AEA and V-bigness imply that $L=\mathcal{O}_{\mathbb{P}}(1)$ is a big and nef line bundle. By the previous lemma, $\mathbb{B}_{+}(L)=\mathbb{T}(L)$, hence

$$
E \text { is V-big } \Longleftrightarrow \pi\left(\mathbb{B}_{+}(L)\right) \neq X \Longleftrightarrow \pi(\mathbb{T}(L)) \neq X \Longleftrightarrow E \text { is AEA. }
$$

Acknowledgments The authors are grateful for the excellent working conditions to the Banff International Research Station, where part of this collaboration took place during the workshop Positivity of Vector Bundles and Linear Series, and to the Hausdorff Research Institute for Mathematics (Bonn) where part of this collaboration did continue during the Junior Trimester Program Algebraic Geometry.

\section{References}

1. Bauer, T., Funke, M.: Weyl and Zariski chambers on K3 surfaces. Forum Math. 24(3), 609-625 (2012)

2. Boucksom, S., Demailly, J.-P., Păun, M., Peternell, T.: The pseudo-effective cone of a compact Kähler manifold and varieties of negative Kodaira dimension. J. Algebr. Geom. 22(2), 201-248 (2013)

3. Brotbek, D.: Projective Varieties with Ample Cotangent Bundle. PhD thesis, Université de Rennes 1 (2011). https://tel.archives-ouvertes.fr/tel-00677065/document 
4. Diverio, S., Rousseau, E.: The exceptional set and the Green-Griffiths locus do not always coincide (2014). arXiv:1302.4756

5. Ein, L., Lazarsfeld, R., Mustaţă, M., Nakamaye, M., Popa, M.: Asymptotic invariants of base loci. Ann. Inst. Fourier (Grenoble) 56(6), 1701-1734 (2006)

6. Ein, L., Lazarsfeld, R., Mustaţă, M., Nakamaye, M., Popa, M.: Restricted volumes and base loci of linear series. Am. J. Math. 131(3), 607-651 (2009)

7. Hartshorne, R.: Ample Subvarieties of Algebraic Varieties, Lecture Notes in Mathematics, vol. 156. Springer, Berlin (1970)

8. Jabbusch, K.: Notions of Positivity for Vector Bundles. PhD thesis, University of Washington. ProQuest LLC, Ann Arbor (2007)

9. Jabbusch, K.: Positivity of cotangent bundles. Mich. Math. J. 58(3), 723-744 (2009)

10. Lang, S.: Hyperbolic and Diophantine analysis. Bull. Am. Math. Soc. (N.S.) 14(2), 159-205 (1986). http://www.ams.org/journals/bull/1986-14-02/S0273-0979-1986-15426-1/home.html

11. Lazarsfeld, R.: Positivity in Algebraic Geometry. I, II. Ergebnisse der Mathematik und ihrer Grenzgebiete. 3. Folge. A Series of Modern Surveys in Mathematics, vol. 48, 49. Springer, Berlin (2004)

12. Lehmann, B.: The movable cone via intersections (2012). arXiv:1111.3928

13. Lesieutre, J.: The diminished base locus is not always closed (2014). arXiv:1212.3738

14. Miyaoka, Y.: Algebraic surfaces with positive indices. In: Ueno, K. (ed.) Classification of Algebraic and Analytic Manifolds (Katata, 1982). Progress in Mathematics, vol. 39, pp. 281-301. Birkhäuser, Boston (1983)

15. Nakamaye, M.: Stable base loci of linear series. Math. Ann. 318(4), 837-847 (2000)

16. Nakayama, N.: Zariski-Decomposition and Abundance. MSJ Memoirs, vol. 14. Mathematical Society of Japan, Tokyo (2004)

17. Urbinati, S.: On the Properties of the Integral Part of Ample and Big Divisors. Master's thesis, Università degli Studi Roma Tre (2007). http://ricerca.mat.uniroma3.it/users/lopez/Tesi-Urbinati.pdf

18. Viehweg, E.: Weak positivity and the additivity of the Kodaira dimension for certain fibre spaces. In: Iitaka, S. (ed.) Algebraic Varieties and Analytic Varieties (Tokyo, 1981). Advanced Studies in Pure Mathematics, vol. 1, pp. 329-353. North-Holland, Amsterdam (1983) 